prophylaxis using fluconazole $400 \mathrm{mg}$ once daily versus amphotericin B lipid complex $2.5 \mathrm{mg} / \mathrm{kg}$ three times per week in adult patients with ALL during the neutropenic nadir who received hyper-CVAD as part of their chemotherapy regimen. Methods This was a retrospective, cohort chart review study conducted in eligible patients with ALL who received a hyperCVAD- based chemotherapy regimen between 1 January 2007 and 31 December 2016 at KAMC, Jeddah. We included patients with ALL aged older than 14 years who completed at least one course of hyper-CVAD and received antifungal prophylaxis. We excluded patients who received the BFM regimen. Data were collected using a hospital information system. The primary endpoint was the incidence of fungal infection, which was assessed using microbiology data and imaging studies for radiological evidence of fungal infections. The secondary endpoints were to assess QTc prolongation in Philadelphia-positive ALL that is associated with fluconazole use in combination with tyrosine kinase inhibitor, and lastly the cost impact based on the type of antifungal prophylaxis used.

Results A total of 105 cycles of hyper-CVAD were reviewed. In 70 cycles, fluconazole was used as antifungal prophylaxis $(n=70)$ and in 35 cycles amphotericin B lipid complex was used $(n=35)$ as antifungal prophylaxis. Microbiologically documented fungal infection was found in two of 70 cycles in the fluconazole group and radiologically documented fungal infection was found in one patient in the fluconazole group. QTc prolongation was observed in 12 cycles. In nine of 12 cycles, events of QTc prolongation were observed during the study, fluconazole was used as antifungal prophylaxis, and patients were on tyrosine kinase inhibitor. In three of 12 cycles, events of QTc prolongation were observed during the study, amphotericin B lipid complex was used as antifungal prophylaxis, and patients were on tyrosine kinase inhibitor.

Conclusion Fluconazole is considered as standard antifungal prophylaxis in patients with ALL with acceptable safety profiles. Fluconazole had comparable efficacy to amphotericin lipid complex. Fluconazole may cause QTc prolongation when used in combination with tyrosine kinase inhibitors and patients need to be monitored more closely when this combination is used in those with Philadelphia-positive ALL.

\section{CONSISTENCY OF CT-KUB RADIATION DOSE AND EXPOSURE PARAMETERS IN KING ABDUL-AZIZ MEDICAL CITY IN JEDDAH: QUALITY ASSESSMENT}

Rahaf Hameed Almoutairi, Shared Hassan Bugis, Zainab Ali Alharbi, Samaher Mohammad Alattas, Ahmad Subahi, Khalid Alshamrani, Shaza Alsharif. Radiology, King Saud bin Abdulaziz University for Health Sciences

\subsection{6/bmjoq-2019-PSF.11}

Background Computed tomography (CT) is a type of medical imaging that uses $\mathrm{x}$-rays to generate cross-sectional images of the body. Although CT improved the limitations in conventional radiography, the effective dose for CT procedures is higher than the dose of a conventional radiographic examination of the same part. For instance, the radiation dose for kidney, ureter, and bladder (KUB) in conventional radiography is $0.7 \mathrm{mSv}$, whereas in the CT scan it is $10 \mathrm{mSv}$. Therefore, patient radiation safety in compliance with American College of Radiology (ACR) standards has to be maintained. In this study, we aimed to evaluate the patient radiation dose delivered in unenhanced CT-KUB examinations using two CT machines at King Abdulaziz Medical City in Jeddah (KAMCJD).

Methods This was a retrospective, cross-sectional study of all patients who had unenhanced CT-KUB examinations in KAMC-JD between 1 January and 30 June 2018. Patient characteristics and radiation parameters were obtained from the hospital information system and pictures archiving and communication system, respectively, and compared with the ACR standards.

Results A total of 264 patients were included in the study: 199 (75.5\%) examinations were performed with machine 1 and $65(24.6 \%)$ were performed with machine 2. Mean age was $48.1 \pm 16.3$ years and $66.7 \%$ of patients were male. Mean body-mass index (BMI) was $29.59 \pm 6.45 \mathrm{~kg} / \mathrm{m} 2$. The mean volume CT dose index (CTDIvol), dose-length product (DLP), time(s), pitch, and effective dose were significantly different in the CT machines with $\mathrm{p}$ values between 0.004 and $<0.001$. No significant differences were found in $\mathrm{kVp}$, slice number, slice thickness, length of cover, and effective dose with use of different types of CT. Both CT machines were within the ACR standard range of $10 \mathrm{mSv}$. There was a positive strong correlation between BMI $\left(29.6 \mathrm{~kg} / \mathrm{m}^{2}\right)$ and the effective dose $(\mathrm{p}<0.001)$.

Conclusion The radiation doses delivered by the two CT scanners in KAMC-JD are compliant with ACR standards. Periodic assessment is recommended every 2 years.

\section{OUTPATIENT FOLLOW-UP IS ASSOCIATED WITH REDUCED EMERGENCY DEPARTMENT VISITS IN PATIENTS WITH SICKLE CELL DISEASE: A RETROSPECTIVE COHORT STUDY FROM RIYADH, SAUDI ARABIA}

Rana Ahmed Saleh, Lama Alhmaly, Ramesh Vishwakarma, Ismat Kamran, Moussab Damlaj, Ahmed Alaskar, Giamal Edin Gmati. Medicine Department, King Abdulaziz Medical City, National Guard Health Affairs

\subsection{6/bmjoq-2019-PSF.12}

Background Pain is the main source of distress in patients with sickle cell disease (SCD). Unmanageable pain, often termed 'crises', may prompt unexpected visits to the emergency department (ED) or other acute healthcare facilities. One study from the Eastern Province in Saudi Arabia reported that $64 \%$ of patients with SCD present to the ED more than three times over a 6-month period. Outpatient follow-up may have a role in preventing ED visits, but this has not been fully explored in Saudi Arabia. This study aimed to assess the relation between outpatient follow-up and future ED visits due to painful crises in patients with SCD.

Methods This retrospective, observational study utilized data from the electronic medical record system at King Abdulaziz Medical City, National Guard Health Affairs in Riyadh. The study included adult patients with SCD who presented at least once to the ED due to a painful crisis between January 2016 and December 2017. The time between ED visits due to painful crises was set as the outcome variable. Cox regression analysis with random effects model (frailty model) was used to determine the effect of outpatient follow-up at 15 days, 30 days, and 90 days on the time to next ED visit. Baseline characteristics and previous history of sickle cell complication were included in the model as confounders.

Results Eighty patients with SCD presented a total of 463 times to the ED due to painful crises. $54 \%$ of these visits 
required admission and resulted in a total length of stay of 1474 hospital days and 49 ICU days. Attendance at a hematology outpatient clinic within 30 days from discharge significantly reduced the hazard of an ED visit due to a painful crisis compared with no follow-up (hazard ratio $0.70,95 \% \mathrm{CI}$ 0.52-0.94, $\mathrm{p}=0.02$ ).

Conclusion Appropriate outpatient referral at discharge may be associated with decreased ED visits. These benchmark results invite further investigation into the effects of outpatient management on preventing painful crises in patients with SCD.

\section{IMPACT OF IMPLEMENTATION OF 'SICKLE CELL DISEASE ACUTE PAINFUL CRISIS CLINICAL PATHWAY' AT KFAFH ON REDUCING THE NUMBER OF ER VISITS, ADMISSION, READMISSION RATES, OPIOID CONSUMPTION, AND COST}

Aseel Jambi, Ahmed Alhartani, Ali Al-Blowi. Pharmacy, King Fahad Armed Forces Hospital

\subsection{6/bmjoq-2019-PSF.13}

Background Vaso-occlusive crisis is the most common complication of sickle cell disease (SCD) in adults, which is the primary reason why these patients seek medical care in emergency departments (EDs). We noticed increasing numbers of ED visits with a painful crisis together with increasing consumption of opioids. Therefore, a clinical pathway for the management of SCD acute painful crisis has been initiated at KFAFH in Jeddah. The main objectives of this clinical pathway management were to unify practice, standardize care, and promote judicious use of opioids at KFAFH. The aims of this study were:

- To reduce the rate of ER visits per patient by more than $50 \%$ within 1 year.

- To decrease the rate of admissions per patient and readmission rates by more than $30 \%$ and $40 \%$, respectively, within 1 year.

- To reduce the consumption of meperidine and tramadol by more than $25 \%$ and $40 \%$, respectively, within 1 year as a secondary outcome.

Methods A clinical pathway for the management of SCD acute painful crisis has been initiated at KFAFH in Jeddah. A total of 374 patients with SCD (aged 12 years or older with isolated painful crisis) was identified by KFAFH Emergency Department registration data. The primary source of patient information was the patient file, ED registration, and chart review for 1 year before (May 2016 to April 2017) implementation of the clinical pathway and 1 year after (May 2017 to April 2018) implementation of the clinical pathway.

Results ED visits:

- The annual rate of ED visits per patient dropped by $75.55 \%$ (45 versus 11.5).

- There was a progressive reduction in the frequency of ED visits over the year.

Admission rate:

- The rate of admissions per patient dropped by $41.87 \%$. Readmission rate:

- The readmission rate declined by $54.51 \%$.
Opioid consumption:

- Consumption of opioids decreased by 33\% for meperidine $100 \mathrm{mg}$ and by $41.6 \%$ for meperidine $50 \mathrm{mg}$. Consumption significantly decreased by $54 \%$ for tramadol capsules and by $33 \%$ for tramadol injection.

Cost saving:

- The cost saved after implementation of the clinical pathway is more than 410,709 SR.

Conclusion Proper pain management for patients with SCD leads to a significant decrease in ED visits, and reduces hospital admissions and readmission rates. Consequently, more than 400,000 SR was saved after the implementation of the pathway. We believe that physicians, nurses, and patient education sessions played a critical role in the success of the clinical pathway.

\section{QUALITY OF LIFE OF PATIENTS POST OPEN CARDIAC SURGERY AT TERTIARY CARE CENTER (2-YEAR RETROSPECTIVE STUDY)}

Danya Adeib Aljafari, Ayman Kurdi, Nizar Althubaiti, Abdulrahman Ayoub. College of Medicine, King Saud University for Health Sciences

\subsection{6/bmjoq-2019-PSF.14}

Background Every minute a patient dies because of acute coronary heart disease in the USA. To prevent death, multiple cardiac procedures are done; however, these procedures do not guarantee a better quality of life. No research has been conducted in Saudi Arabia and the Gulf region regarding quality of life of patients post cardiac surgery. The aim of this study was to explore the quality of life of patients who underwent open cardiac surgeries in King Faisal Cardiac Center-King Abdulaziz Medical City-Western Region of Saudi Arabia during 2015-2017. The study assessed the quality of life of surviving patients and identified the association between demographic characteristics and quality of life domains.

Methods A cross-sectional retrospective study was done at King Faisal Cardiac Center. A convenience sample was used by identifying all patients who underwent open cardiac surgery between June 2015 and May 2017. 163 patients were eligible for our study, of whom 94 were valid for analysis. These patients were sent the SF-36 questionnaire, which is proven to evaluate quality of life. The survey is divided into eight major domains, which were reported as mean scores. Correlation was done using the Mann-Whitney $U$ test, using a cutoff $\mathrm{p}$ value of 0.05 .

Results The study reported that subscales of role limitations due to emotional problems and physical health were the lowest of the eight domains. However, the emotional well-being and the pain subscales were the highest of them all. We found that cardiovascular diseases, compared with other comorbidities, had the biggest effect on lowering quality of life.

Conclusion The results of the present study indicate that quality of life of patients who underwent cardiac surgery is affected negatively. The immediate post-operative duration is a vital factor that impacts long-term quality of life; therefore, hospital services need to increase their efforts regarding this critical period. Further studies should be done to explore the reasons behind these findings. 